\title{
Automated Dual-Exposure Technique to Extend the Dynamic Range of Flat-Panel Detectors Used in Small-Animal Cone-Beam Micro-CT
}

\author{
Alejandro Sisniega, Student Member IEEE, Juan J. Vaquero, Member IEEE, Mónica Abella, Irina Vidal Migallón, \\ Eduardo Lage and Manuel Desco, Member IEEE
}

\begin{abstract}
This work presents an approach to extend the dynamic range of $\mathrm{X}$ ray flat panel detectors for cone beam micro CT by using two different acquisitions of the same sample, taken at two different $X$ ray photon fluxes with the same $X$ ray beam peak energy and filtration. Photon flux for the first scan is chosen as the maximum possible value not saturating the detector in the low attenuation areas. The second exposure level is calculated from the data acquired in the first exposure, assuming that the detector response to the incoming radiation is linear. To combine both datasets, we model the response of a detector pixel, as well as the overall gain of the detector. Each pixel of each dataset is weighted according to its value. Pixels whose value lies within the high gain region of the detector receive the higher weights. The weighted Joint Probability Density Function (JPDF) is obtained by assuming that each dataset follows an independent Gaussian distribution. The final pixel value is calculated by means of a maximum likelihood estimation performed on the JPDF.

The algorithm has been tested imaging two different phantoms on a small animal cone beam CT. We have performed comparative experiments using the proposed dual exposure technique and a regular single exposure acquisition, following a fully automated procedure. In both cases the dose delivered to the sample was the same. Image quality was assessed from the image noise level and the presence of artifacts on the reconstructed slices, at different dose levels. The dual exposure data show higher signal to noise ratio. Also, image artifacts are noticeably reduced in the dual exposure dataset. Since the proposed technique enhance the quality of the data extending the dynamic range of the detector without increasing the delivered dose, it is particularly suitable to image samples which contain both low and high attenuating regions. Furthermore, the extension of the technique to use more than two exposure levels is straightforward.
\end{abstract}

\section{INTRODUCTION}

$\mathrm{C}$ ONE-BEAM micro-CT (CBCT) is usually employed in small-animal imaging as a stand-alone technique, or to provide complementary anatomical information for other

This work is partially funded by the CD-TEAM Project, (CENIT, Ministerio de Industria), projects TEC2007-64731 and TEC2008-06715-C02-01, RETICRECAVA network and European Project FP7-201792.

Authors are with the Unidad de Cirugía y Medicina Experimental, Hospital General Universitario Gregorio Marañón, Madrid, Spain.

E-mail: desco@hggm.es molecular imaging modalities such as PET or SPECT. However, Flat-Panel (FP) semiconductor detectors commonly used in CBCT suffer from a limited dynamic range, which compromise image quality when a sample has both low and high density materials (like the brain inside the skull, or metallic probes inside the body). The problem may arise in any CT scanner, whenever the dynamic range of the real scene exceeds that of the detector.

Several approaches have been proposed to address this issue, most of them originated from the optical imaging field (i.e., photography and video). Some of the aforementioned techniques are based on the acquisition of several images, each one with a different exposure to the incident radiation. Afterwards, the acquired images are combined to obtain a new High-Dynamic-Range (HDR) image. The combination algorithm to obtain the value of a pixel of the HDR image can be as simple as the selection of the best sample from the original data [1] or a more elaborated process which takes advantage from all the information contained in the data acquired [2]. The use of this kind of techniques to extend the dynamic range of FP detectors in CBCT has been previously reported [3]. However, the exposure level selection and the combination algorithm are not fully developed and the performance of the method has not been compared with that achieved by conventional scanning protocols.

Other methods to extend the dynamic range of imaging systems involve hardware modifications or special detector designs. Some of these approaches include the use of nonconventional pixel design [4], multiple sensors inside a single pixel [5] or spatially varying pixel sensitivity [6].

We introduce here an automatic dual-exposure technique based on a weighting scheme that takes into account both detector and sample properties.

\section{MATERIAL AND MethodS}

\section{A. Data Acquisition and Processing}

In order to obtain the HDR data, the sample is scanned twice. The first scan uses the maximum X-ray flux that does not saturate the detector in low attenuation areas. The X-ray 
source anode current for the second scan is calculated from the average histogram of all the angular projections obtained in the first scan, removing those pixels whose value is closed to the flood data. Assuming a linear detector response [7], the new current value is obtained by shifting the most populated part of the histogram, to fit within the high-gain region.

The combination of the two datasets is based on the algorithm reported in [2], adapted to the particular features of $\mathrm{X}$-ray FP detectors.

The value for pixel $j$ and acquisition $i(i=1,2)$ is modeled by the equation

$$
Y_{i j}=\frac{A_{i}}{e^{(\mu x)_{j}}}+N_{i j}
$$

Where $A_{i}$ stands for the ratio between the first and the $i$ th acquisition currents (exposures), and $N_{i j}$ represents an additive noise term that includes noise sources derived from both the acquisition statistics and the quantification process in the digital detector. The second noise term affects in a stronger way to those pixels whose value is closer to the weakest detectable signal, thus being significantly reduced in the high current dataset.

We assume that the pixel noise of each acquisition follows an independent Gaussian distribution. The new pixel value is obtained as the result of a Maximum-Likelihood calculation based on the joint probability density function (JPDF). This JPDF is estimated by weighting the raw pixel values as a function of their position in the detector response curve. Those pixels within the high-gain region receive a higher weight.

Since the defined JPDF is Gaussian, the objective function to minimize is given by

$$
O=\sum_{i} w_{i j}\left(Y_{i j}-\frac{A_{i}}{e^{(\mu x)_{j}}}\right)
$$

Where $w_{i j}$ stands for the weight assigned to pixel $j$ and acquisition $i$.

Thus, the expression to calculate the estimated HDR pixel value is:

$$
\left(\frac{1}{e^{(\mu x)_{j}}}\right)=\frac{\sum_{i} w_{i j} A_{i} Y_{i j}}{\sum_{i} w_{i j} A_{i}^{2}}
$$

\section{B. Performance Evaluation}

We have tested the algorithm on data obtained from phantoms scanned with an Argus PET-CT system [8] (Sedecal, Torrejon, Spain).

Two different phantoms have been scanned. The first one consists of three plastic rods (Teflon, Delrin and Nylon), surrounded by a layer of copper $0.3 \mathrm{~mm}$ thick. A regular hypodermic needle is placed approximately in the center of the phantom.
The second phantom consists of a bar pattern made of ten layers of copper with different thicknesses, ranging from 0.6 $\mathrm{mm}$ to $1.5 \mathrm{~mm}$. The step size is $0.1 \mathrm{~mm}$.

The tomographic images were reconstructed using a modified FDK algorithm adapted to the scanner geometry [8].

Quality of the reconstructed slices has been assessed by measuring the signal to noise ratio in the different regions.

Each scan was performed twice; a first time using the dualexposure technique and a second time using a conventional scanning protocol, delivering the same dose to the sample in both cases.

The increase in detector dynamic range has been assessed on planar images of the described bar pattern. Several profiles along the pattern have also been measured.

\section{PRELIMINARY ReSUlts}

The signal to noise ratio was evaluated from three scans, acquired with different dose levels (Table 1). The highest delivered dose is represented as $100 \%$. The X-ray beam peak energy was $45 \mathrm{kV}$ with $1 \mathrm{~mm} \mathrm{Al}$ added filtration.

TABLE I. SNR VALUES FOR THE DIFFERENT ACQUIRED DATASETS

\begin{tabular}{|c|c|c|c|c|}
\hline \multirow{2}{*}{ Dose } & $\begin{array}{c}\text { Scan } \\
\text { protocol }\end{array}$ & $\begin{array}{c}\text { SNR } \\
\text { PTFE }\end{array}$ & $\begin{array}{c}\text { SNR } \\
\text { Delrin }\end{array}$ & $\begin{array}{c}\text { SNR } \\
\text { Nylon }\end{array}$ \\
\hline \multirow{2}{*}{$100 \%$} & Dual & 11.35 & 12.69 & 10.90 \\
\cline { 2 - 5 } & Single & 9.99 & 10.47 & 7.59 \\
\hline \multirow{2}{*}{$67 \%$} & Dual & 10.58 & 10.40 & 8.73 \\
\cline { 2 - 5 } & Single & 5.98 & 8.44 & 9 \\
\hline
\end{tabular}

The reconstructed slices show strong dark strikes on the single-exposure data. These artifacts mainly derive from photon starvation on the detector when the X-ray beam crosses a thick region of high attenuation material, such as copper. Using the dual-exposure technique proposed, these artifacts are noticeably reduced.

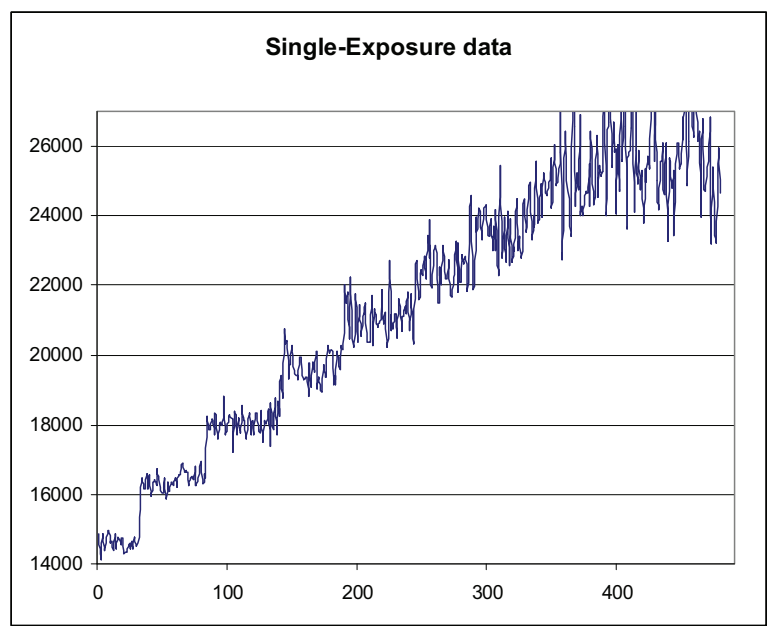

Fig. 1. Profile plot across the bar pattern phantom, for the single exposure protocol. 


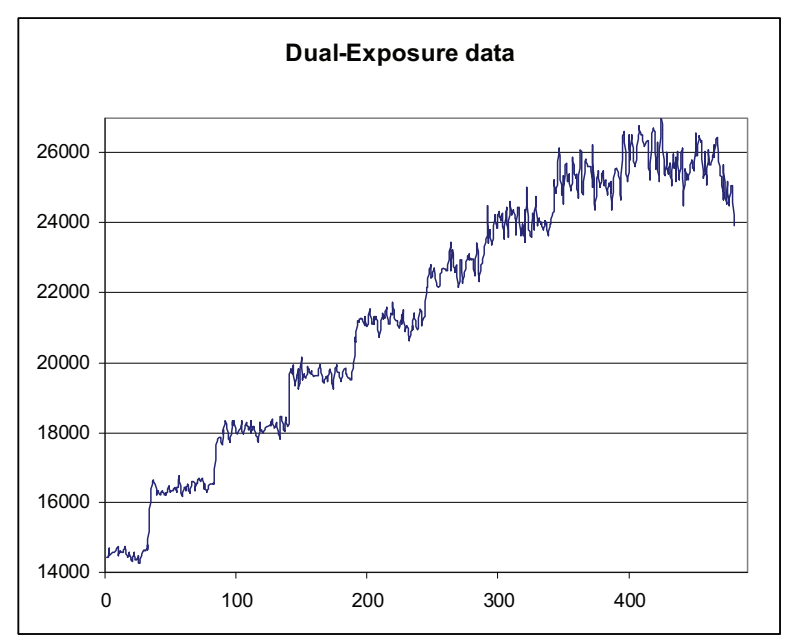

Fig. 2. Profile plot across the bar pattern phantom, for the dual exposure protocol.

Ring artifacts, typical of flat-panel detectors, are more conspicuous in the single-exposure protocol.

The copper bars phantom was scanned using $40 \mathrm{kV}$ X-ray beam peak energy and $1 \mathrm{~mm} \mathrm{Al}$ filter with the dual and single exposure protocols, again delivering the same dose in both cases. The signal inside the high attenuation areas is masked by noise on the single exposure data. When applying the dual exposure technique, the noise level is significantly reduced, enhancing the signal to noise ratio. Profiles across the bar pattern, for both acquisitions are plotted in fig. 1 and fig. 2 .

\section{REFERENCES}

[1] B.C. Madden. Extended intensity range imaging. Technical report, GRASP Lab. University of Pennsylvania, 1993.

[2] M.A. Robertson, et.al. Dynamic range improvement through multiple exposures. Int. Conf. on Image Processing, 1999.

[3] P. Sukovic, et.al. A method for extending the dynamic range of Flat Panel imagers for use in Cone-Beam Computed Tomography. Nuclear Science Symposium Conference Record, vol. 3, 1647-1651, 2001.

[4] T. Lule, et.al. Design and fabrication of a High-Dynamic-Range image sensor in TFA technology. IEEE Journal of Solid-State Circuits, vol.34, 5, 704-711, 1999.

[5] R.A. Street. High dynamic range segmented pixel array. US Patent $5,099,505,1992$.

[6] S. Nayar, et.al. High dynamic range imaging: spatially varying pixel exposures. IEEE Conference on Computer Vision and Pattern Recognition, vol.1, 472-479, 2000.

[7] Kim et.al. Use of Flat-Panel detector for microtomography: A feasibility study for small animal imaging. IEEE Trans. on Nuclear Science, vol. 52, 1, 193-198, 2005.

[8] J.J. Vaquero, et.al. Assessment of a new high-performance small-animal Xray tomography. IEEE Trans. on Nuclear Science, vol. 55, 3, 898-905, 2008 . 